\title{
rTMS modulates precuneus-hippocampal subregion circuit in subjective cognitive decline
}

Jiu Chen ( $\nabla$ ericcst@aliyun.com )

Nanjing Medical University

\section{Guanjie Hu}

Affiliated Brain Hospital of Nanjing Medical University

Chen Xue

Affiliated Brain Hospital of Nanjing Medical University

Wenzhang Qi

Affiliated Brain Hospital of Nanjing Medical University

Wenwen Xu

Affiliated Brain Hospital of Nanjing Medical University

Shanshan Chen

Affiliated Brain Hospital of Nanjing Medical University

Jiang Rao

Affiliated Brain Hospital of Nanjing Medical University

Wan Liu

Affiliated Brain Hospital of Nanjing Medical Universtiy

Fuquan Zhang

Affiliated Brain Hospital of Nanjing Medical University

Xiangrong Zhang ( $\nabla$ drxrz@hotmail.com )

Affiliated Brain Hospital of Nanjing Medical University

\section{Research}

Keywords: episodic memory, functional connectivity, hippocampal-subregion, rTMS, SCD

Posted Date: August 1st, 2020

DOI: https://doi.org/10.21203/rs.3.rs-47474/v1

License: (c) (i) This work is licensed under a Creative Commons Attribution 4.0 International License.

Read Full License 


\section{Abstract}

Background: Hippocampal subregions (HIPsub) and their network connectivities are considered to be abnormal in subjective cognitive decline (SCD). This study aimed to identify whether repetitive transcranial magnetic stimulation (rTMS) could ameliorate the HIPsub network connectivity by modulating one node of HIPsub network in SCD subjects.

Methods: In the first cohort (38 SCD and 55 healthy controls), we analyzed three HIPsub (i.e., hippocampal emotional, cognitive, and perceptual regions: HIPe, HIPc, and HIPp) functional connectivity to identify HIPsub network connectivity alterations that relate to SCD. And we further applied a support vector machine (SVM) approach using the alterations to identify how well this could distinguish SCD from CN. In the second cohort (13 SCD), rTMS with 5-day of once-daily for 2 weeks was used to modulate the altered HIPsub network connectivity in a sham-controlled design.

Results: SCD subjects showed differently altered patterns of HIPsub network connectivity compared to controls. The SVM classifier showed that the abnormalities had a high power to discriminate SCD from CN, with an ACC of $86.0 \%$, an AUC of $92.9 \%, 83.8 \%$ sensitivity, and $89.1 \%$ specificity. Restoration of HIPC connectivity with left parahippocampa gyrus and HIPp connectivity with left middle temporal gyrus corresponded to amelioration of episodic memory in SCD patients after 2 weeks of rTMS.

Conclusion: rTMS can restore the posterior hippocampus connectivity by modulating the precuneus in SCD subjects. Correction of breakdown in HIPc and HIPp related to cognitive and perceptual processing can simultaneously ameliorate episodic memory in SCD subjects. Thus, these findings suggest that rTMS manipulation of precuneus-hippocampal circuit may prevent the disease progression by improving memory as the earliest at-risk state of $A D$ in clinical trials.

Trial registration: CCTR, ChiCTR2000034533. Registered 9 July 2020 - Retrospectively registered, http://www.chictr.org.cn

\section{Background}

Subjective cognitive decline (SCD), who has self-reported persistent memory decline while cognitive performance remains within the normal range, is well-known considered as the earliest at-risk state of Alzheimer's disease (AD) even before amnestic mild cognitive impairment (MCI) [1-6]. Hippocampus, a hallmark of $A D$ [7-9], is thought as one of the earliest brain regions to present with pathology that causes memory decline. A great number of studies have consistently reported hippocampal atrophy in SCD subjects [3, 10-12]. However, little is known about whether hippocampal network connectivity is also dysfunctional in SCD subjects, especially the hippocampal subregions networks. Therefore, our lack of understanding of their pathophysiology hampers the development of new interventions to prevent the clinical progression of SCD to MCl/AD. 
Neuroimaging evidences have consistently indicated a functional heterogeneity in hippocampus subregions (HIPsub) [13-15]. A recent neuroimaging meta-analytic study has defined the left hippocampus as consisting of the anterior emotional region (HIPe), the middle cognitive region (HIPc), and the posterior perceptual region (HIPp) based on its neurofunctional topography [13]. Furthermore, several studies have indicated that HIPsub have a selective topography of pathological involvement in preclinical $A D[12,15,16]$. Recently, several studies have reported that SCD present the structural and functional alterations of hippocampus $[3,10-12,16]$. Converging evidences suggest that SCD may present differently altered patterns of HIPsub network connectivity. Consequently, there is growing interest in answering the question how the dysfunctions in HIPsub network connectivity can be restored.

Neuromodulation techniques, including repetitive transcranial magnetic stimulation (rTMS), afford an opportunity to address this challenge by modulating intrinsic connectivity networks. Recently, rTMS has been broadly applied to study the changes across cortical networks [17-20]. Local stimulation of an accessible network node can be transmitted across synapses means to remote interconnected nodes with high spatial specificity $[18,21,22]$. Therefore, this approach can allow to establish a causal link between the applied stimulation and the observed changes in HIPsub network connectivity.

Recently, a neuromodulation study has verified that rTMS targeting the parietal cortex can improve hippocampal connectivity networks, and simultaneously improve associative memory performance in healthy individuals [20]. A recent $\mathrm{MCl}$ neuromodulation study applies rTMS to promote the improvement of episodic memory by targeting the precuneus [23]. The precuneus is well-known thought as a remote interconnected node of hippocampal intrinsic connectivity networks $[20,24]$ and a critical vulnerability area for the episodic memory deficit observed in early $\operatorname{AD}[25,26]$. Based on above-mentioned evidences, it is reasonable to speculate that the altered HIPsub network connectivity can be causally effected by rTMS modulation upon the precuneus in the HIPsub network in SCD subjects.

In this study, we propose a strategy to empirically discover a HIPsub pathological circuit related to SCD using a pattern classification approach (SVM: support vector machine), and subsequently, in a targeted manner, this circuit was experimentally manipulated to assess causal links in a separate cohort. We hypothesized that SCD subjects would display differently altered patterns of HIPsub network connectivity. And we further hypothesized that breakdown in HIPsub circuit related to episodic memory processing could be restored by rTMS modulation upon the precuneus in the HIPsub network in SCD subjects. Figure $\mathbf{S} 1$ shows the data analysis pipeline conducted in this study.

\section{Materials And Methods}

\section{Participants}

Data used in this study were obtained from our in-home database: Nanjing Brain Hospital-Alzheimer's Disease Spectrum Neuroimaging Project (NBH-ADsnp) (Nanjing, China), which is constantly being updated. Relevant information of NBH-ADsnp is summarized in SI Methods S.2. 


\section{Network discovery of altered HIPsub related to SCD}

A total of 99 elderly individuals participated in this study. Of the subjects, we excluded 2 healthy controls (CN) and 4 SCD subjects due to excessive head movement (see quality assurance section below), and incomplete or missing MRI data. The final analyses included $55 \mathrm{CN}$ and $38 \mathrm{SCD}$ eligible subjects. SI Methods S. 2 shows detailed inclusion and exclusion criteria.

\section{Network validation of altered HIPsub related to SCD with rTMS}

A total of 20 SCD subjects participated in the clinical trial (No. ChiCTR2000034533) in this study from the NBH-ADsnp database.

We used rTMS (or sham) with 5-day of once-daily to modulate the precuneus of SCD subjects for 2 weeks in a sham-controlled design. Clinical measures, neuropsychological assessments, and MRI data were collected at baseline (pre-rTMS or sham intervention) and at the end of 2 weeks of rTMS or sham. A total of 20 SCD subjects were enrolled in the study, of which 16 SCD subjects were randomly divided into real rTMS (8 SCD) or sham (8 SCD), and 13 SCD subjects (8 SCD for real rTMS, 5 SCD for sham rTMS) completed the trial of 2 weeks of rTMS.

\section{Neuropsychological assessment}

Neuropsychological assessments are summarized in SI MethodsS.3. This study performed a standardized clinical interview and comprehensive neuropsychological assessment to evaluate general cognitive function, executive function, information processing speed, episodic memory, and visuo-spatial function.

\section{MRI data acquisition}

Detailed MRI data acquisition parameters in NBH-ADsnp are summarized in SI MethodsS.4.

\section{fMRI data preprocessing}

In this study, MATLAB2015b and DPABI software [27] were used to preprocess all fMRI data. The image processing procedure was performed as described in a previous study [28] and is summarized in $\boldsymbol{S I}$

Methods S.5.

Quality assurance (QA) 


\section{Brain atrophy effect}

Given that significant hippocampal GM atrophies in SCD subjects have been reported [12, 29], the anatomical differences between groups may affect these differences on the FCs of HIPsub. To clarify this issue, we computed global intracranial volumes (ITV) based on native GM, WM, and CSF in CN and SCD subjects by using in-home MATLAB codes. Furthermore, we used ITV as an additional covariate when general linear model (GLM) analysis is used to investigate the differences on the network connectivity of HIPsub between $\mathrm{CN}$ and SCD subjects.

\section{Head motion effect}

In this study, we used three approaches to control the head motion effect both at the individual and at group levels. Firstly, we excluded SCD subjects with excessive head motion (cumulative translation or rotation $>3.0 \mathrm{~mm}$ or $3.0^{\circ}$ ). Then we used a Friston 24-parameter model to regress out head motion effects from the realigned data [30]. Secondly, we performed a 'scrubbing' procedure to scrub frames (volumes) with an excessively high whole-brain root mean square (RMS) signal change over time in the preprocessed fMRI data for each individual [31-33]. Furthermore, we regressed out all volumes with a framewise displacement (FD) greater than $0.2 \mathrm{~mm}$ as nuisance covariates, and discarded any scan with $50 \%$ of volumes removed as described in a previous study [34]. Overall, we excluded $1 \mathrm{CN}$ because of excessive head movement. No significant differences were observed in the head motion parameters between qualified CN and SCD subjects (Table 1).

\section{Strict multiple comparison correction strategy}

To ensure the reproducibility, test-retest reliability, and replicability on the fMRI metrics, we performed a strict multiple comparison correction [35], that is, statistical maps were thresholded using the permutation test with Threshold-Free Cluster Enhancement (TFCE) [36] and the false discovery rate (FDR), as implemented in DPABI [27]. For cluster-extent permutation tests, voxel thresholds of two-tailed $p<0.02$ ( $Z$ $>2.3$ ) were set. Finally, we set a two-tailed $p<0.05$ threshold (1,000 permutations in FDR evaluation).

\section{Definition of hippocampal subregions}

Our definition of HIPsub referred to recent studies from Robinson et al. [13] and Bai et al.[37], who used coactivation-based parcellation to reveal a subspecialization in the hippocampus by a data-driven method. We only selected the left HIPsub as regions of interest (ROI) (Fig. S2) based on the recent study published by Bai and colleagues [37]. The left hippocampus was defined as three subregions (HIPe, HIPc, and HIPp).

\section{Functional connectivity analyses}


First, we extracted the average time courses for all voxels within each HIPsub as the reference time course. Second, we performed voxelwise cross-correlation analysis between the averaged time courses of all voxels within the seed HIPsub region and each voxel in the remainder of the whole brain within the group-specific GM mask. Finally, we performed a Fisher's z-transform analysis to enhance the normality of the correlation coefficients.

\section{rTMS protocol}

rTMS was used to stimulate the precuneus of all aMCl participants using a Magstim Rapid2 magnetic stimulator with a 70-mm figure-8-shaped coil. We used the Pz site of the 10-20 electroencephalogram system to locate the precuneus, and the tip of the intersection of the two coil loops was placed at the $\mathrm{Pz}$ site to stimulate the precuneus [23].

rTMS was applied, using trains of 1000 stimuli at a frequency of $20 \mathrm{~Hz}$ and at an intensity of $100 \%$ of the motor threshold (MT). We defined the MT as the lowest intensity producing motor evoked potentials of greater than $50 \mu \mathrm{V}$ in at least 5 out of 10 trials in the relaxed first dorsal interosseous (FDI) muscle of the contralateral (right) hand [38]. Participants received 25 sessions of either rTMS or sham stimulation over the precuneus. Each daily stimulation session consisted of a stimulation of $42 \mathrm{~s}$ duration with an interval of $28 \mathrm{~s}$. The entire session lasted approximately 30 minutes each daily. We performed the sham rTMS blocks with the coil held close to the precuneus, but angled away.

\section{TMS protocol adverse events}

The participants did not report any adverse events during the rTMS trial.

\section{Statistical Analysis}

\section{Demographics and neuropsychological data}

We performed two-sample t-test and chi-square tests to assess differences in demographic data, clinical, cognitive performance, ITV, and head rotation parameters between SCD and CN subjects $(p<0.05)$.

\section{Network discovery of altered HIPsub related to SCD}

To characterize the HIPsub network FC patterns at a group level, we performed a random-effect analysis using one-sample $t$-tests in the spatial maps of FC in CN and SCD subjects with a stringently threshold of $p<0.001$ using the permutation test with TFCE and the family-wise error (FWE) correction together with a cluster extent $\mathrm{k}>100$ voxels $\left(2700 \mathrm{~mm}^{3}\right)$.

We performed GLM analysis to investigate the differences in the FCs of HIPsub between SCD subjects and CN before rTMS treatment after controlling for age, sex, education, ITV, and mean FD (TFCE-FDR- 
corrected $p<0.05$ and cluster size $>405 \mathrm{~mm}^{3}$ ). Then we made masks based on brain regions showing differences in the FCs of HIPsub in SCD compared to CN. These masks were used for the analysis of prev.s. post-rTMS (pre-sham- v.s. post-sham-rTMS) fMRI data from study \#2 (i.e., network validation of altered HIPsub related to SCD). These findings identified network connectivity of altered HIPsub related to $S C D$, which can explain the changes that are related to SCD during rTMS treatment.

\section{Pattern classification based on the altered HIPsub GM and FC}

To further identify GM and network connectivity of altered HIPsub as closely related to SCD patients, we applied a support vector machine (SVM) approach using the alterations in the identified ROls as a biomarker to test how well this could distinguish SCD patients from CN subjects. A leave-one-out crossvalidation (LOOCV) strategy was used to assess the generalization of this SVM classifier and to assess its accuracy, sensitivity, and specificity. These findings identified GM and network connectivity of altered HIPsub related to SCD patients, which can explain the changes that are related to SCD during rTMS treatment.

\section{Network validation of altered HIPsub related to SCD with rTMS}

To empirically validate altered HIPsub network connectivity related to SCD, we used paired t-tests to calculate the changes in network FC of HIPsub pre- v.s. post-rTMS (or pre-sham- v.s. post-sham-rTMS) in SCD subjects after controlling for age, sex, education, and GM.

\section{Sham v.s. real rTMS comparison}

Of the 13 SCD subjects with full clinical assessments, usable SMRI, and fMRI scan data at baseline and 2 weeks of post-rTMS (or sham), 8 subjects had been randomized to real rTMS and 5 subjects received the sham rTMS. We performed a two-sample t-test to investigate the differences in the changes in FC of HIPsub between pre-post real rTMS and pre-post sham rTMS. Pre-real-rTMS (or sham-rTMS) maps were subtracted from post-real-rTMS (or sham-rTMS) maps to generate maps of FC changes for each subject.

\section{Non-parametric statistics}

To improve the statistical power with a low sample size, we performed a re-sampling method of stationary 10,000 bootstrap samplings to obtain significance in demographic data, clinical characteristics, cognitive performance, and FC of HIPsub between baseline assessment and at 2 weeks of post-rTMS (sham rTMS) for all statistical analyses (i.e., chi-square test, two-sample t-test, Pearson correlation, and paired-sample t-test).

\section{Study approval}

This study was approved by the responsible Human Participants Ethics Committee of the Affiliated Brain Hospital of Nanjing Medical University (No. 2018-KY010-01 and No. 2020-KY010-02) (Nanjing, China). 


\section{Results}

\section{Demographics, and clinical and cognitive function characteristics}

As shown in Table 1, compared with CN, SCD subjects did not show no any significant differences in age, gender, education, general cognitive function (i.e., MMSE, MoCA, MDRS scores), and multimodal cognitive function (i.e., episodic memory, information processing speed, executive function, visuospatial function) (all $p>0.05,10000$ bootstraps) when showed higher HAMD and SCD-Q (all $p<0.05,10000$ bootstraps).

\section{Network discovery of altered HIPsub related to SCD}

As shown in Figure 1-3 and Figure S3, SCD subjects displayed distinctly differently altered patterns of HIPsub network connectivity compared to controls.

As shown in Figure 4A and Table S3, in the HIPe network, compared with CN, SCD subjects displayed reduced FC in right CERpos, increased FC in FusG.L, Insula.L and PHG.L $\left(P_{\text {TFCE-FDR }}<0.05\right.$, cluster size $>$ $405 \mathrm{~mm}^{3}$ ). As shown in Figure 4B and Table S3, in the HIPc network, compared with CN, SCD displayed reduced FC in IFGorb.R, increased FC in PHG.L $\left(P_{\text {TFCE-FDR }}<0.05\right.$, cluster size $\left.>405 \mathrm{~mm}^{3}\right)$. As shown in Figure $4 \mathrm{C}$ and Table S3, in the HIPp network, compared with CN, SCD displayed reduced FC in MFG.L, increased FC in Insula.L, bilateral MTG, PreCUN.L $\left(P_{\text {TFCE-FDR }}<0.05\right.$, cluster size $\left.>405 \mathrm{~mm}^{3}\right)$. All results were controlled for age, sex, education, ITV, and FD.

\section{Classification of aMCl patients based on the altered HIPsub GM volumes and functional connectivities in SCD patients}

The SVM classifier's classification accuracy was $86.0 \%$. As shown in Figure 5, the SVM classifier's receiver operating characteristic (ROC) curve shows a high power to discriminate SCD patients from CN on an individual subject basis, with an AUC of $92.9 \%, 83.8 \%$ sensitivity, and $89.1 \%$ specificity.

\section{Network validation of altered HIPsub related to SCD with rTMS}

Changes of HIPsub FC pre v.s. post-rTMS (or sham rTMS)

As shown in Figure 6A, in the HIPc network, compared with pre-rTMS, SCD subjects showed significantly reduced FC in PHG.L at 2 weeks of post-rTMS ( $p<0.05,10000$ bootstraps). As shown in Figure 6B, in the 
HIPp network, compared with pre-rTMS, SCD subjects showed significantly reduced FC FC in MTG.L at 2 weeks of post-rTMS ( $p<0.05,10000$ bootstraps). No differences were found in the HIPe, HIPc and HIPp connectivity pre- v.s. post-sham rTMS.

\section{Changes of episodic memory pre- v.s. post-rTMS (or sham rTMS)}

As shown in Figure 7, SCD subjects showed improvement of episodic memory (AVLT) after 2 weeks of real rTMS treatment ( $p<0.001,10000$ bootstraps) while no differences were observed after 2 weeks of sham TMS treatment ( $p>0.05,10000$ bootstraps).

\section{Discussion}

To our knowledge, this study was the first to answer questions that the dysfunctions in posterior hippocampus (i.e., HIPc and HIPp) network connectivity could be causally restored by rTMS modulation upon the precuneus in SCD subjects. It further suggests that the precuneus-HIPsub circuit may be a potential target circuit to prevent the clinical progression of SCD to MCI/AD in therapeutic trials.

This study showed that SCD subjects presented differently altered patterns of HIPsub network connectivity compared to controls, which supports the notion that HIPsub involves in functional heterogeneity [13-15]. The SVM classifier showed that the abnormalities in brain memory networks had a high power to discriminate SCD from CN on an individual subject basis, with an ACC of $86.0 \%$, an AUC of 92.9\%, 83.8\% sensitivity, and 89.1\% specificity, which suggests that this study identifies a HIPsub pathological circuit related to SCD. The HIPe network connectivity in SCD subjects is predominantly abnormal in the brain regions involved in many aspects of emotional processing, including the cerebellum [39, 40], anterior insula [39, 41], parahippocampa gyrus [39, 42], and fusiform gyrus [43]. Indeed, SCD subjects are characterized by self-reported concerns regarding their memory decline [44], which significantly increases the risk of $A D$ [5]. Based on above-mentioned evidence, it suggests that SCD subjects exist the abnormal network connectivity associated with emotional processing. In particular, SCD subjects showed altered HIPC and HIPp network connectivity in these brain regions involved in memory processing: the input and integration of sensory perception spatial information, visual object recognition memory, and the formation of episodic memory [15, 45-47]. Based on the processing theory of memory system, memory formation requires two neruopathways: occipito-temporal visual object processing pathway (the "what" stream) $[47,48]$ and parieto-temporal visuospatial pathway (the "where" stream) $[49,50]$. Therefore, our results suggest that although SCD self-reported memory declines within the normal range, there are abnormalities in the networks associated with memory processing. That is, abnormalities in brain memory networks may precede the onset of clinical symptoms in SCD subjects.

More especially, the most fascinating finding was that rTMS modulation upon the precuneus for 2 weeks could restore HIPc connectivity with left parahippocampa gyrus and HIPp connectivity with left middle temporal gyrus accompanied by improvement of episodic memory. Indeed, numerous studies have consistently indicated that the precuneus is a node belonging to hippocampal intrinsic connectivity networks [15, 20, 24], a key node for episodic memory deficits observed in early $\operatorname{AD}[25,26]$, and a 
vulnerable region for the progress of $\mathrm{MCI}$ to $\mathrm{AD}$ [23]. Local rTMS stimulating accessible network nodes can be transmitted across synapses to distant but highly spatially specific interconnected nodes $[18,21$, 22]. Therefore, it is reasonable to speculate that the effects of rTMS may propagate in the hippocampus through synaptic transmission in the precuneus-HIPsub pathway. Furthermore, this study only showed that rTMS modulation could restore the dysfunctions in posterior hippocampus (i.e., HIPc and HIPp) network connectivity related to memory processing but did not the dysfunctions in anterior hippocampus (i.e., $\mathrm{HIPe}$ ) network connectivity related to emotional processing. It suggests that the precuneus-HIPsub pathway is an ideal target circuit for tailored rTMS intervention to improve episodic memory decline in SCD.

\section{Limitations}

First, a relatively small sample size was used to validate the effects of rTMS on the HIPsub pathological circuit related to SCD in this study. According to the general rTMS clinical trials, a single clinical symptom indicator was used as the clinical efficacy criteria. In this case, our sample size is indeed relatively small. However, this study combined clinical response with functional connectivity as the efficacy criteria, so it can be speculated that our conclusions are still reliable. Second, our findings suggested a potential target circuit the restoring the dysfunctions in HIPc and HIPp network connectivity, but this study did not find a target circuit for the restoration of HIPe network connectivity. In the future, a large sample size is needed to explore the therapeutic pathways of anterior hippocampus network connectivity.

\section{Conclusions}

This study provides a novel experimental evidence on correction of breakdown in posterior hippocampus (HIPc and HIPp) related to cognitive and perceptual processing by modulating the precuneus in SCD subjects. Furthermore, rTMS manipulation may prevent the disease progression by improving memory as the earliest at-risk state of $A D$ in clinical treatment trials. It further suggests that the precuneus-HIPsub circuit may be used as a useful target circuit for SCD subjects to design rationale strategies for therapeutic trials.

\section{Abbreviations}

HIPsub: hippocampal subregions; SCD: subjective cognitive decline; rTMS: repetitive transcranial magnetic stimulation; HIPe: hippocampal emotional region; HIPc: hippocampal cognitive region; HIPp: hippocampal perceptual region; AD: Alzheimer's disease; $\mathrm{MCl}$ : amnestic mild cognitive impairment; NBHADsnp: Nanjing Brain Hospital-Alzheimer's Disease Spectrum Neuroimaging Project; CN: healthy controls; ITV: intracranial volumes; GLM: general linear model; RMS: root mean square; FD: framewise displacement; TFCE: threshold-free cluster enhancement; FDR: false discovery rate; ROI: regions of interest; MT: motor threshold; FWE: family-wise error; CEREpos.R: right cerebellum posterior lobe; FusG.L: left fusiform gyrus; PHG.L: left parahippocampa gyrus; IFGorb.R: right inferior frontal gyrus, orbital part; 
MTG.R\ right middle temporal gyrus; MTG.L: left middle temporal gyrus; MFG.L: left medial frontal gyrus; PreCUN.L: left Precuneus.

\section{Declarations}

\section{Author contributions}

JC and XZ: designed the study. GH,CX, WQ, WM, WX, SC, JR, and WL: collected the data. FZ and JC: analyzed the data and prepared the manuscript.

\section{Acknowledgments}

The authors sincerely thank these doctors from department of radiology and rehabilitation for their help with acquisition of behavioural data and MRI data, and for taking care of clinical and rTMS data in this study.

\section{Funding}

This study was supported by the National Key Research and Development Program of China (No. 2018YFC1314300), the National Natural Science Foundation of China (No. 81701675; 81971255); the Key Project supported by Medical Science and technology development Foundation, Nanjing Department of Health (No. JQX18005); the Cooperative Research Project of Southeast University-Nanjing Medical University (No. 2018DN0031); the Key Research and Development Plan (Social Development) Project of Jiangsu Province (No. BE2018608; BE2019610); Jiangsu Provincial Medical Talent project (ZDRCA2016075); the Innovation and Entrepreneurship Training Program for College Students in Jiangsu Province (No.201810312061X; 201910312035Z); and Key Scientific Research Projects of Colleges and Universities in Henan Province (No:18A190003).

\section{Availability of data and materials}

The dataset generated and analyzed in the current study is available from the corresponding author on reasonable request.

\section{Ethics approval and consent to participate}

This study was approved by the responsible Human Participants Ethics Committee of the Affiliated Brain Hospital of Nanjing Medical University (No. 2018-KY010-01 and No. 2020-KY010-02) (Nanjing, China). Written informed consent was received from participants prior to inclusion in the study.

\section{Consent for publication}

Not applicable.

\section{Competing interests}


The authors have declared that no conflict of interest exists

\section{Supplementary material}

Please see the supplementary material.

\section{References}

1. van der Flier WM, van Buchem MA, Weverling-Rijnsburger AW, Mutsaers ER, Bollen EL, AdmiraalBehloul $F$, et al. Memory complaints in patients with normal cognition are associated with smaller hippocampal volumes. J Neurol. 2004; 251:671-675.

2. Stewart R, Dufouil C, Godin O, Ritchie K, Maillard P, Delcroix N, et al. Neuroimaging correlates of subjective memory deficits in a community population. Neurology. 2008; 70:1601-1607.

3. Striepens N, Scheef L, Wind A, Popp J, Spottke A, Cooper-Mahkorn D, et al. Volume loss of the medial temporal lobe structures in subjective memory impairment. Dement Geriatr Cogn Disord. 2010; 29:7581.

4. Scheef L, Spottke A, Daerr M, Joe A, Striepens N, Kolsch H, et al. Glucose metabolism, gray matter structure, and memory decline in subjective memory impairment. Neurology. 2012; 79:1332-1339.

5. Jessen $F$, Wolfsgruber $S$, Wiese $B$, Bickel $H$, Mosch $E$, Kaduszkiewicz $H$, et al. AD dementia risk in late $\mathrm{MCl}$, in early $\mathrm{MCl}$, and in subjective memory impairment. Alzheimers Dement. 2014; 10:76-83.

6. Buckley RF, Maruff P, Ames D, Bourgeat P, Martins RN, Masters CL, et al. Subjective memory decline predicts greater rates of clinical progression in preclinical Alzheimer's disease. Alzheimers Dement. 2016; 12:796-804.

7. Sperling RA, Aisen PS, Beckett LA, Bennett DA, Craft S, Fagan AM, et al. Toward defining the preclinical stages of Alzheimer's disease: recommendations from the National Institute on AgingAlzheimer's Association workgroups on diagnostic guidelines for Alzheimer's disease. Alzheimers Dement. 2011; 7:280-292.

8. Simon SS, Yokomizo JE and Bottino CM. Cognitive intervention in amnestic Mild Cognitive Impairment: a systematic review. Neurosci Biobehav Rev. 2012; 36:1163-1178.

9. Bourrelier J, Kubicki A, Rouaud O, Crognier L and Mourey F. Mental Rotation as an Indicator of Motor Representation in Patients with Mild Cognitive Impairment. Front Aging Neurosci. 2015; 7:238.

10. Jessen F, Feyen L, Freymann K, Tepest R, Maier W, Heun R, et al. Volume reduction of the entorhinal cortex in subjective memory impairment. Neurobiol Aging. 2006; 27:1751-1756.

11. Meiberth D, Scheef L, Wolfsgruber S, Boecker H, Block W, Traber F, et al. Cortical thinning in individuals with subjective memory impairment. J Alzheimers Dis. 2015; 45:139-146.

12. Perrotin A, de Flores R, Lamberton F, Poisnel G, La Joie R, de la Sayette V, et al. Hippocampal Subfield Volumetry and 3D Surface Mapping in Subjective Cognitive Decline. J Alzheimers Dis. 2015; 48 Suppl 1:S141-150. 
13. Robinson JL, Barron DS, Kirby LA, Bottenhorn KL, Hill AC, Murphy JE, et al. Neurofunctional topography of the human hippocampus. Hum Brain Mapp. 2015; 36:5018-5037.

14. Davachi L. Item, context and relational episodic encoding in humans. Curr Opin Neurobiol. 2006; 16:693-700.

15. Chen J, Duan X, Shu H, Wang Z, Long Z, Liu D, et al. Differential contributions of subregions of medial temporal lobe to memory system in amnestic mild cognitive impairment: insights from fMRI study. Sci Rep. 2016; 6:26148.

16. Liang L, Zhao L, Wei Y, Mai W, Duan G, Su J, et al. Structural and Functional Hippocampal Changes in Subjective Cognitive Decline From the Community. Front Aging Neurosci. 2020; 12:64.

17. Rastogi A, Cash R, Dunlop K, Vesia M, Kucyi A, Ghahremani A, et al. Modulation of cognitive cerebello-cerebral functional connectivity by lateral cerebellar continuous theta burst stimulation. Neuroimage. 2017; 158:48-57.

18. Eldaief MC, Halko MA, Buckner RL and Pascual-Leone A. Transcranial magnetic stimulation modulates the brain's intrinsic activity in a frequency-dependent manner. Proc Natl Acad Sci U S A. $2011 ; 108: 21229-21234$.

19. Siddiqi SH, Taylor SF, Cooke D, Pascual-Leone A, George MS and Fox MD. Distinct Symptom-Specific Treatment Targets for Circuit-Based Neuromodulation. Am J Psychiatry. 2020; 177:435-446.

20. Wang JX, Rogers LM, Gross EZ, Ryals AJ, Dokucu ME, Brandstatt KL, et al. Targeted enhancement of cortical-hippocampal brain networks and associative memory. Science. 2014; 345:1054-1057.

21. Ruff CC, Bestmann S, Blankenburg F, Bjoertomt O, Josephs O, Weiskopf N, et al. Distinct causal influences of parietal versus frontal areas on human visual cortex: evidence from concurrent TMSfMRI. Cereb Cortex. 2008; 18:817-827.

22. Halko MA, Farzan F, Eldaief MC, Schmahmann JD and Pascual-Leone A. Intermittent theta-burst stimulation of the lateral cerebellum increases functional connectivity of the default network. J Neurosci. 2014; 34:12049-12056.

23. Koch G, Bonni S, Pellicciari MC, Casula EP, Mancini M, Esposito R, et al. Transcranial magnetic stimulation of the precuneus enhances memory and neural activity in prodromal Alzheimer's disease. Neuroimage. 2018; 169:302-311.

24. Kahn I, Andrews-Hanna JR, Vincent JL, Snyder AZ and Buckner RL. Distinct cortical anatomy linked to subregions of the medial temporal lobe revealed by intrinsic functional connectivity. $J$ Neurophysiol. 2008; 100:129-139.

25. Buckner RL, Andrews-Hanna JR and Schacter DL. The brain's default network: anatomy, function, and relevance to disease. Ann N Y Acad Sci. 2008; 1124:1-38.

26. Raichle ME, MacLeod AM, Snyder AZ, Powers WJ, Gusnard DA and Shulman GL. A default mode of brain function. Proc Natl Acad Sci U S A. 2001; 98:676-682.

27. Yan CG, Wang XD, Zuo XN and Zang YF. DPABI: Data Processing \& Analysis for (Resting-State) Brain Imaging. Neuroinformatics. 2016; 14:339-351. 
28. Yan CG, Craddock RC, Zuo XN, Zang YF and Milham MP. Standardizing the intrinsic brain: towards robust measurement of inter-individual variation in 1000 functional connectomes. Neuroimage. 2013; 80:246-262.

29. Caillaud M, Hudon C, Boller B, Brambati S, Duchesne S, Lorrain D, et al. Evidence of a Relation Between Hippocampal Volume, White Matter Hyperintensities, and Cognition in Subjective Cognitive Decline and Mild Cognitive Impairment. J Gerontol B Psychol Sci Soc Sci. 2019.

30. Friston KJ, Williams S, Howard R, Frackowiak RS and Turner R. Movement-related effects in fMRI time-series. Magn Reson Med. 1996; 35:346-355.

31. Sheline YI, Raichle ME, Snyder AZ, Morris JC, Head D, Wang S, et al. Amyloid plaques disrupt resting state default mode network connectivity in cognitively normal elderly. Biol Psychiatry. 2010; 67:584587.

32. Power JD, Barnes KA, Snyder AZ, Schlaggar BL and Petersen SE. Spurious but systematic correlations in functional connectivity MRI networks arise from subject motion. Neuroimage. 2012; 59:2142-2154.

33. Van Dijk KR, Sabuncu MR and Buckner RL. The influence of head motion on intrinsic functional connectivity MRI. Neuroimage. 2012; 59:431-438.

34. Brady RO, Jr., Gonsalvez I, Lee I, Ongur D, Seidman LJ, Schmahmann JD, et al. Cerebellar-Prefrontal Network Connectivity and Negative Symptoms in Schizophrenia. Am J Psychiatry. 2019:appiajp201818040429.

35. Chen X, Lu B and Yan CG. Reproducibility of R-fMRI metrics on the impact of different strategies for multiple comparison correction and sample sizes. Hum Brain Mapp. 2018; 39:300-318.

36. Smith SM and Nichols TE. Threshold-free cluster enhancement: addressing problems of smoothing, threshold dependence and localisation in cluster inference. Neuroimage. 2009; 44:83-98.

37. Bai T, Wei Q, Xie W, Wang A, Wang J, Ji GJ, et al. Hippocampal-subregion functional alterations associated with antidepressant effects and cognitive impairments of electroconvulsive therapy. Psychol Med. 2019; 49:1357-1364.

38. Rossini PM, Burke D, Chen R, Cohen LG, Daskalakis Z, Di lorio R, et al. Non-invasive electrical and magnetic stimulation of the brain, spinal cord, roots and peripheral nerves: Basic principles and procedures for routine clinical and research application. An updated report from an I.F.C.N. Committee. Clin Neurophysiol. 2015; 126:1071-1107.

39. Lindquist KA, Wager TD, Kober H, Bliss-Moreau E and Barrett LF. The brain basis of emotion: a metaanalytic review. Behav Brain Sci. 2012; 35:121-143.

40. Adamaszek M, D'Agata F, Ferrucci R, Habas C, Keulen S, Kirkby KC, et al. Consensus Paper: Cerebellum and Emotion. Cerebellum. 2017; 16:552-576.

41. Zhang Y, Zhou W, Wang S, Zhou Q, Wang H, Zhang B, et al. The Roles of Subdivisions of Human Insula in Emotion Perception and Auditory Processing. Cereb Cortex. 2019; 29:517-528.

42. McDonald AJ and Mott DD. Functional neuroanatomy of amygdalohippocampal interconnections and their role in learning and memory. J Neurosci Res. 2017; 95:797-820. 
43. Marshall CR, Hardy CJD, Russell LL, Bond RL, Sivasathiaseelan H, Greaves C, et al. The functional neuroanatomy of emotion processing in frontotemporal dementias. Brain. 2019; 142:2873-2887.

44. Jessen F, Amariglio RE, van Boxtel M, Breteler M, Ceccaldi M, Chetelat G, et al. A conceptual framework for research on subjective cognitive decline in preclinical Alzheimer's disease. Alzheimers Dement. 2014; 10:844-852.

45. Alvarado MC and Bachevalier J. Comparison of the effects of damage to the perirhinal and parahippocampal cortex on transverse patterning and location memory in rhesus macaques. $J$ Neurosci. 2005; 25:1599-1609.

46. Staresina BP, Duncan KD and Davachi L. Perirhinal and parahippocampal cortices differentially contribute to later recollection of object- and scene-related event details. J Neurosci. 2011;31:87398747.

47. Eichenbaum $\mathrm{H}$, Yonelinas AP and Ranganath $\mathrm{C}$. The medial temporal lobe and recognition memory. Annu Rev Neurosci. 2007; 30:123-152.

48. Lavenex P, Suzuki WA and Amaral DG. Perirhinal and parahippocampal cortices of the macaque monkey: Intrinsic projections and interconnections. J Comp Neurol. 2004; 472:371-394.

49. Suzuki WA and Amaral DG. Perirhinal and parahippocampal cortices of the macaque monkey: cortical afferents. J Comp Neurol. 1994; 350:497-533.

50. Kravitz DJ, Saleem KS, Baker Cl and Mishkin M. A new neural framework for visuospatial processing. Nat Rev Neurosci. 2011; 12:217-230.

\section{Tables}

Table 1. Demographics, clinical measures, and head rotation parameters of SCD subjects and CN 


\begin{tabular}{|c|c|c|c|}
\hline \multirow{2}{*}{\multicolumn{2}{|c|}{ Items }} & $\mathrm{CN}$ & SCD \\
\hline & & $\mathrm{n}=55$ & $\mathrm{n}=38$ \\
\hline \multicolumn{2}{|l|}{ Age (years) } & $62.91(5.94)$ & $65.84(7.73)$ \\
\hline \multicolumn{2}{|l|}{ Gender (male/female) } & $23 / 32$ & $8 / 30$ \\
\hline \multicolumn{2}{|l|}{ Education level (years) } & $12.51(2.51)$ & $12.22(2.72)$ \\
\hline \multicolumn{2}{|l|}{ MMSE } & $28.58(1.43)$ & $28.32(2.63)$ \\
\hline \multicolumn{2}{|l|}{ MoCA } & $25.05(2.42)$ & 24.92(1.79) \\
\hline \multicolumn{2}{|l|}{ MDRS } & $141.46(2.33)$ & 140.37(3.05) \\
\hline \multicolumn{2}{|l|}{ HAMD } & $1.82(2.26)$ & $3.92(3.17)^{a}$ \\
\hline \multicolumn{2}{|l|}{ SCD-Q } & $3.55(1.50)$ & $6.51(0.90)^{\mathrm{a}}$ \\
\hline \multicolumn{2}{|l|}{ ITV } & 1130.24(114.65) & $1083.55(109.21)^{\mathrm{a}}$ \\
\hline \multicolumn{4}{|l|}{ Episodic memory tests } \\
\hline \multirow[t]{2}{*}{ AVLT-IR } & raw score & $19.15(4.36)$ & $18.66(4.22)$ \\
\hline & $Z$ score & $0.35(0.94)$ & $0.25(0.91)$ \\
\hline \multirow[t]{2}{*}{ AVLT-5min-DR } & raw score & $6.35(2.20)$ & $6.26(1.90)$ \\
\hline & $Z$ score & $0.34(0.93)$ & $0.31(0.80)$ \\
\hline \multirow[t]{2}{*}{ AVLT-20min-DR } & raw score & $6.30(1.94)$ & $6.32(2.12)$ \\
\hline & $Z$ score & $0.40(0.73)$ & $0.41(0.80)$ \\
\hline AVLT-total & raw score & $31.79(7.61)$ & $31.24(7.39)$ \\
\hline \multicolumn{4}{|c|}{ Composite $\mathrm{Z}$ scores of each cognitive domain } \\
\hline \multicolumn{2}{|l|}{ Episodic memory } & $0.27(0.53)$ & $0.34(0.59)$ \\
\hline \multicolumn{2}{|l|}{ Information processing speed } & $0.27(0.67)$ & $0.18(0.71)$ \\
\hline \multicolumn{2}{|l|}{ Executive function } & $0.27(0.48)$ & $0.30(0.57)$ \\
\hline \multicolumn{2}{|l|}{ Visuospatial function } & $0.17(0.66)$ & $0.26(0.50)$ \\
\hline \multicolumn{4}{|l|}{ Head rotation parameters } \\
\hline \multicolumn{2}{|l|}{ FD_VanDijk } & $0.05(0.03)$ & $0.04(0.03)$ \\
\hline \multicolumn{2}{|l|}{ FD_Power } & $0.18(0.08)$ & $0.16(0.09)$ \\
\hline \multicolumn{2}{|l|}{ FD_Jenkinson } & $0.09(0.04)$ & $0.09(0.05)$ \\
\hline
\end{tabular}

Data are presented as the mean (standard deviation, SD). Abbreviations: CN, healthy controls; SCD, subjective cognitive decline; MMSE, Mini Mental State Examination; MoCA, Montreal Cognitive Assessment; MDRS, Mattis Dementia Rating Scale; HAMD, Hamilton Depression Scale; SCDQ, Subjective Cognitive Decline-Questionnaire; ITV, intracranial volume; AVLT-IR, Auditory Verbal Learning Test - immediate recall; AVLT-5minDR, Auditory Verbal Learning Test - 5-minute delayed recall; AVLT-20min-DR, Auditory Verbal Learning Test - 20-minute delayed recall; FD, framewise displacement. ${ }^{a}$ Significant differences were found between CN and SCD subjects. MMSE, MoCA, and MDRS are displayed as raw scores. This study used the composite Z scores to indicate the level of each cognitive domain. Note, to improve the statistical power, this study used a re-sampling method of stationary bootstrap (10,000 bootstrap samplings) to obtain significance.

\section{Figures}




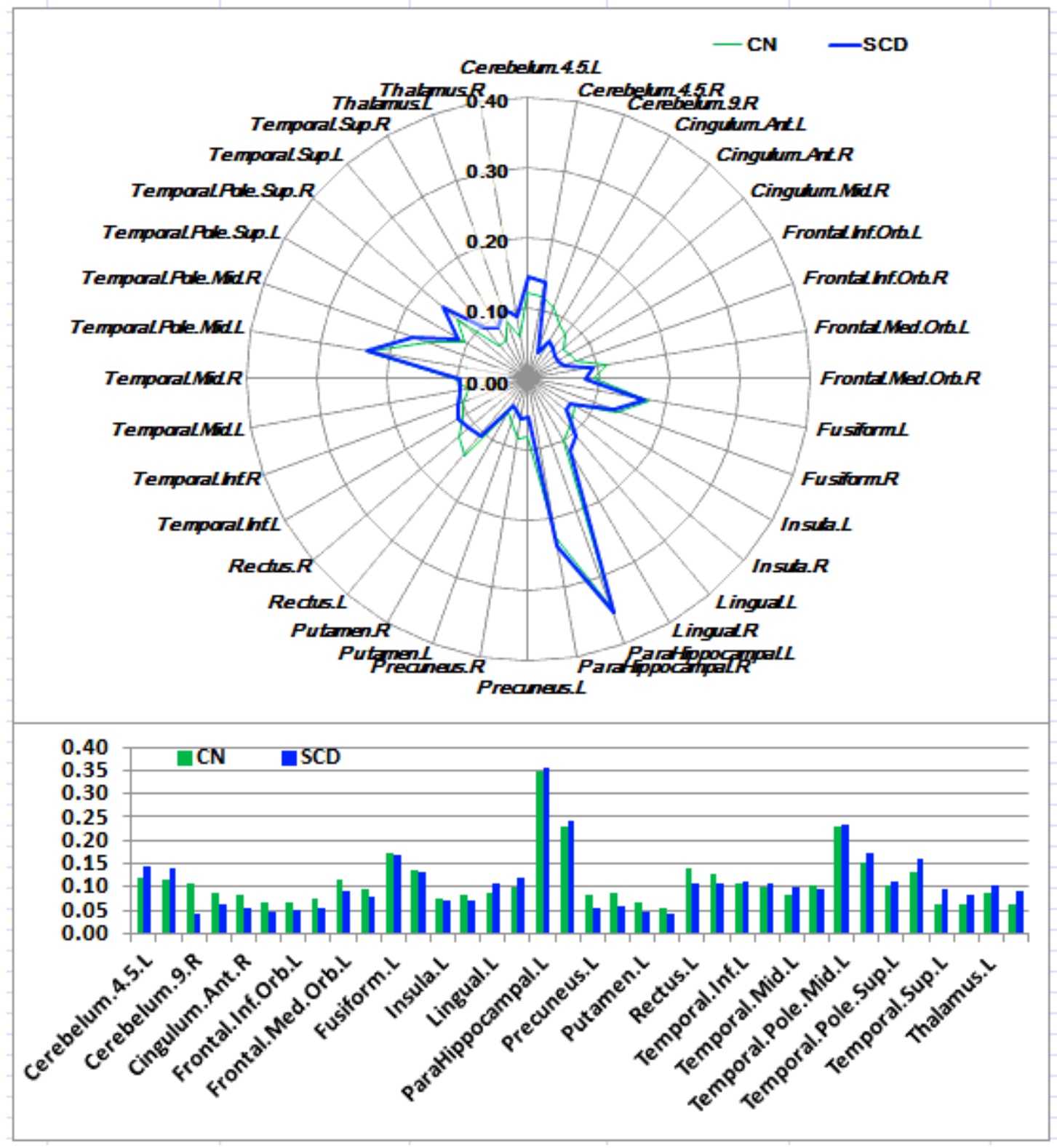

Figure 1

Schematic polar plot and bar chart depicting distinct functional connectivity patterns of HIPe seeds with target regions of interest (ROI) distributed across the whole brain among $\mathrm{CN}$ and SCD subjects. The concentric circles depict parameter estimates representing the connectivity strength. Note that the functional connectivity data are only extracted from the brain regions corresponding to Fig. S3. Abbreviations: $\mathrm{CN}$, healthy controls; $\mathrm{SCD}$, subjective cognitive decline; HIPe, hippocampal emotional region; $\mathrm{ROI}$, region of interest. 


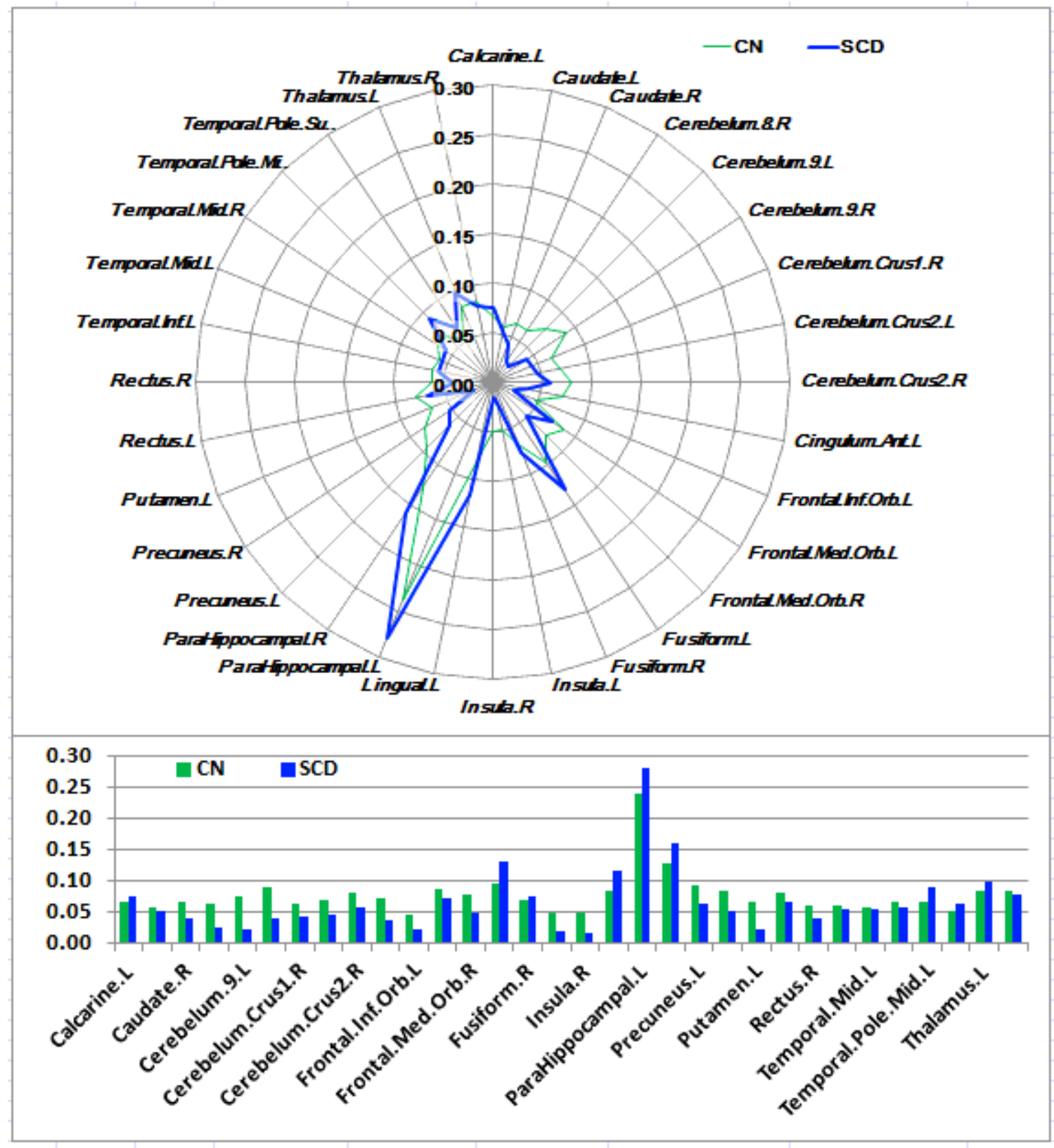

Figure 2

Schematic polar plot and bar chart depicting distinct functional connectivity patterns of HIPc seeds with target regions of interest (ROI) distributed across the whole brain among $\mathrm{CN}$ and SCD subjects. The concentric circles depict parameter estimates representing the connectivity strength. Note that the functional connectivity data are only extracted from the brain regions corresponding to Fig. S3. Abbreviations: $\mathrm{CN}$, healthy controls; $\mathrm{SCD}$, subjective cognitive decline; $\mathrm{HIPc}$, hippocampal cognitive region; $\mathrm{ROI}$, region of interest. 


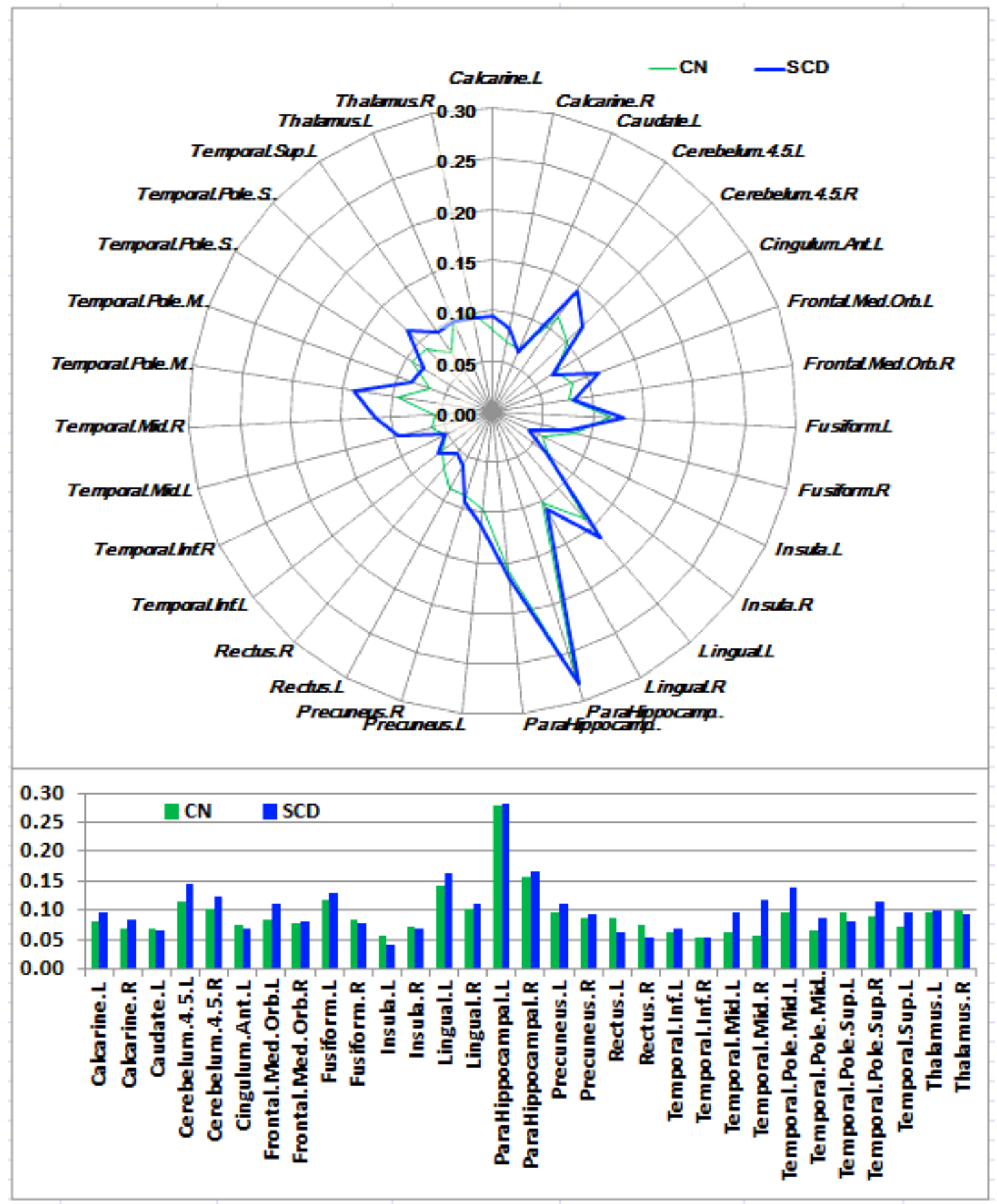

Figure 3

Schematic polar plot and bar chart depicting distinct functional connectivity patterns of HIPp seeds with target regions of interest (ROI) distributed across the whole brain among $\mathrm{CN}$ and SCD subjects. The concentric circles depict parameter estimates representing the connectivity strength. Note that the functional connectivity data are only extracted from the brain regions corresponding to Fig. S3. Abbreviations: $\mathrm{CN}$, healthy controls; SCD, subjective cognitive decline; HIPp, hippocampal perceptual region; $\mathrm{ROI}$, region of interest. 


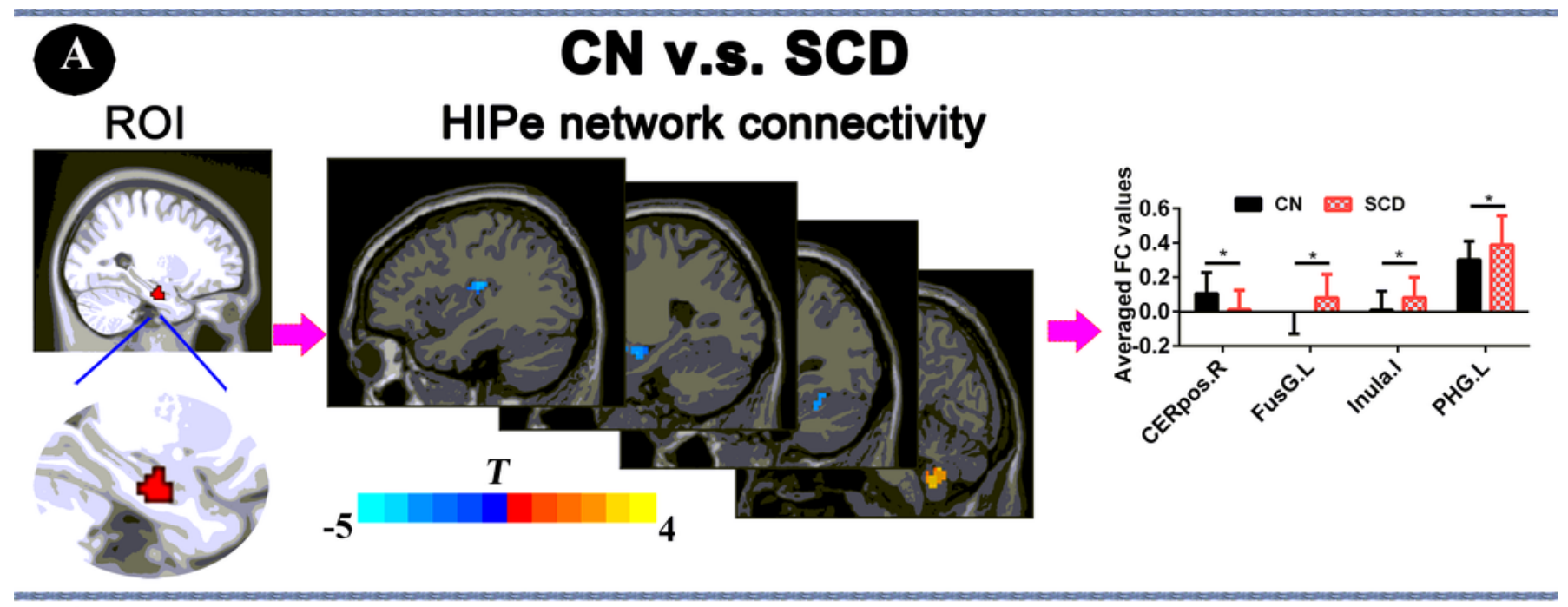

B

ROI

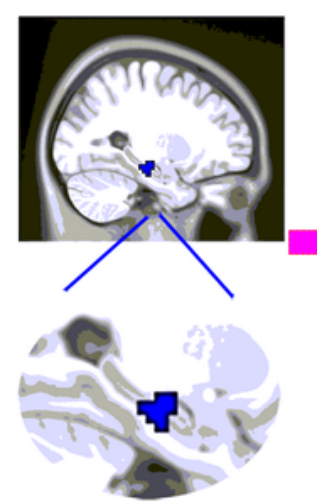

HIPc network connectivity
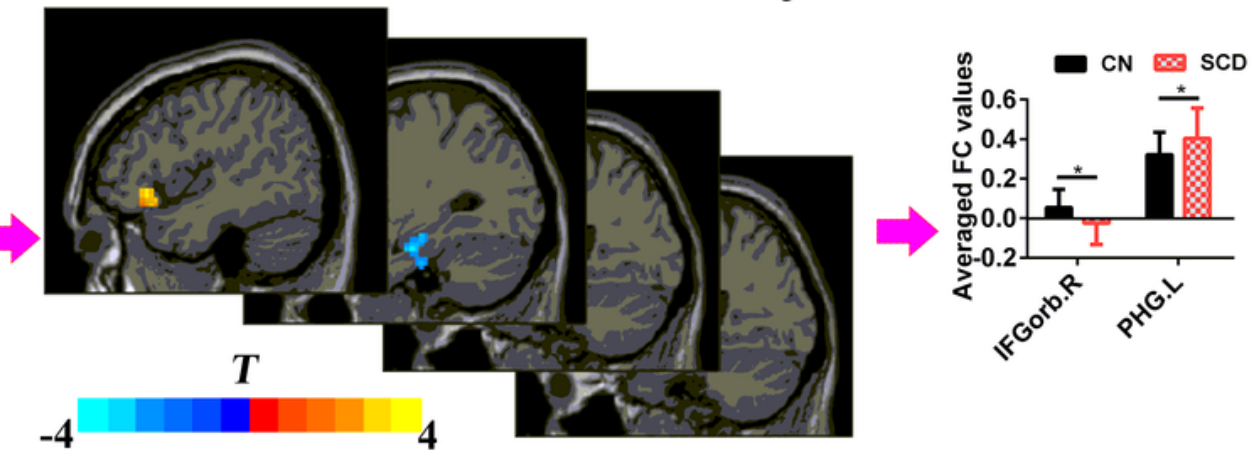

C

\section{ROI}
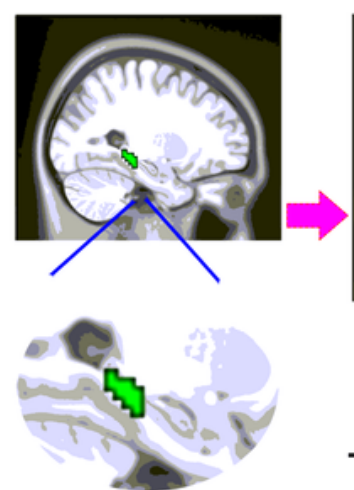

\section{HIPp network connectivity}
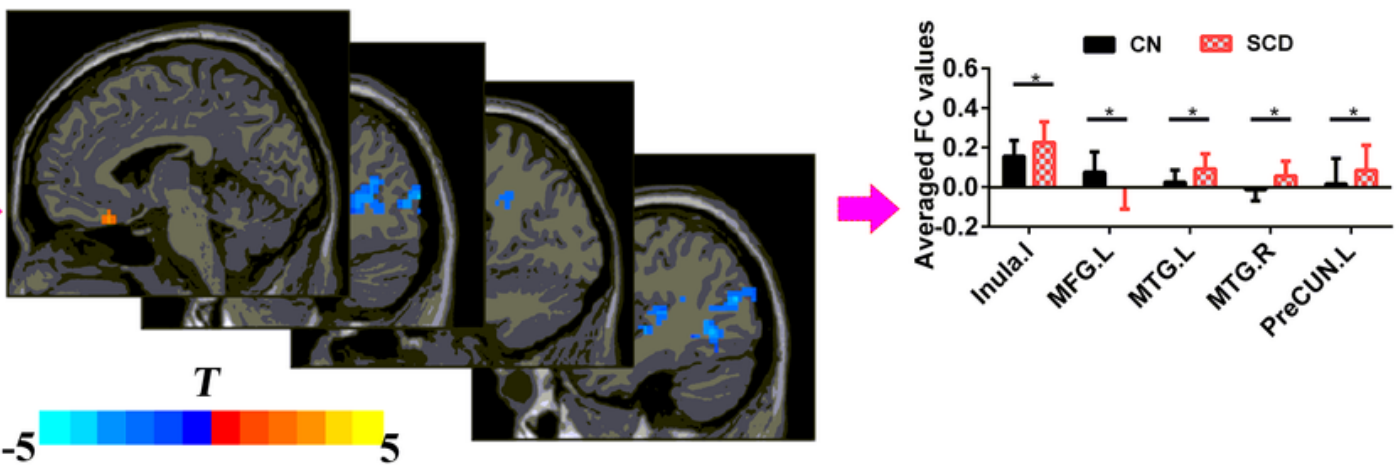

Figure 4

Differences in HIPsub functional connectivity between SCD subjects and CN before rTMS treatment after controlling for age, sex, education, ITV, and FD ( $p<0.05$, TFCE-FDR correction, cluster size $>405 \mathrm{~mm} 3$. (A) HIPe-subregion and brain different regions of the HIPe functional connectivity between $\mathrm{CN}$ and SCD subjects. Bar chart showing the quantitative comparison of functional connectivity in these regions. (B) HIPc-subregion and brain different regions of the HIPc functional connectivity between CN and SCD 
subjects. Bar chart showing the quantitative comparison of functional connectivity in these regions. (C) HIPp-subregion and brain different regions of the HIPp functional connectivity between CN and SCD subjects. Bar chart showing the quantitative comparison of functional connectivity in these regions. * PTFCE-FDR<0.05. Abbreviations: CN, healthy controls; SCD, subjective cognitive decline; HIPe, hippocampal emotional region; HIPc, hippocampal cognitive region; HIPp, hippocampal perceptual region; TFCE, threshold-free cluster enhancement; FDR, false discovery rate; ITV, Intracranial volume; FD, framewise displacement; CEREpos.R, right cerebellum posterior lobe; FusG.L, left fusiform gyrus; PHG.L, left parahippocampa gyrus; IFGorb.R, right inferior frontal gyrus, orbital part; MTG.R, right middle temporal gyrus; MTG.L, left middle temporal gyrus; MFG.L, left medial frontal gyrus; PreCUN.L, left Precuneus. 


\section{CN vs. SCD ROC Curve}

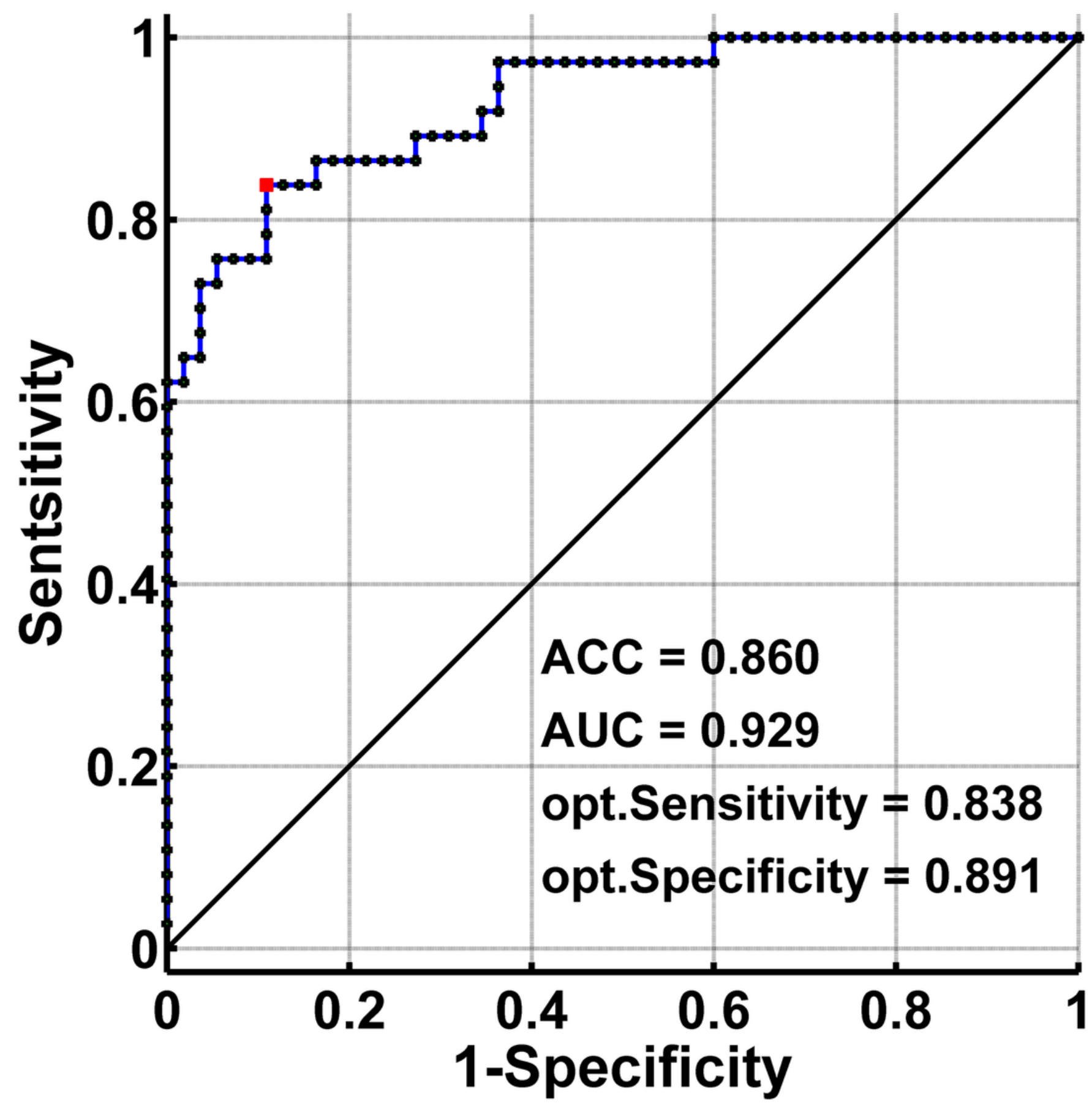

Figure 5

Classification of individuals as SCD versus CN by MRI-based "classifier". ROC curve showing the classification power in MRI-based "classifier" of SCD from CN. Note: the values of ACC, AUC, sensitivity, and specificity in lower right of the figure present the optimum values under the optimum combined index score (red piont). Abbreviations: $\mathrm{SCD}$, subjective cognitive decline; $\mathrm{CN}$, healthy controls; AUC, area under the ROC curve; ACC, accuracy; Opt, optimum; ROC, receiver operating characteristic; MRI, magnetic resonance imaging. 


\section{A \\ 2 weeks of rTMS}
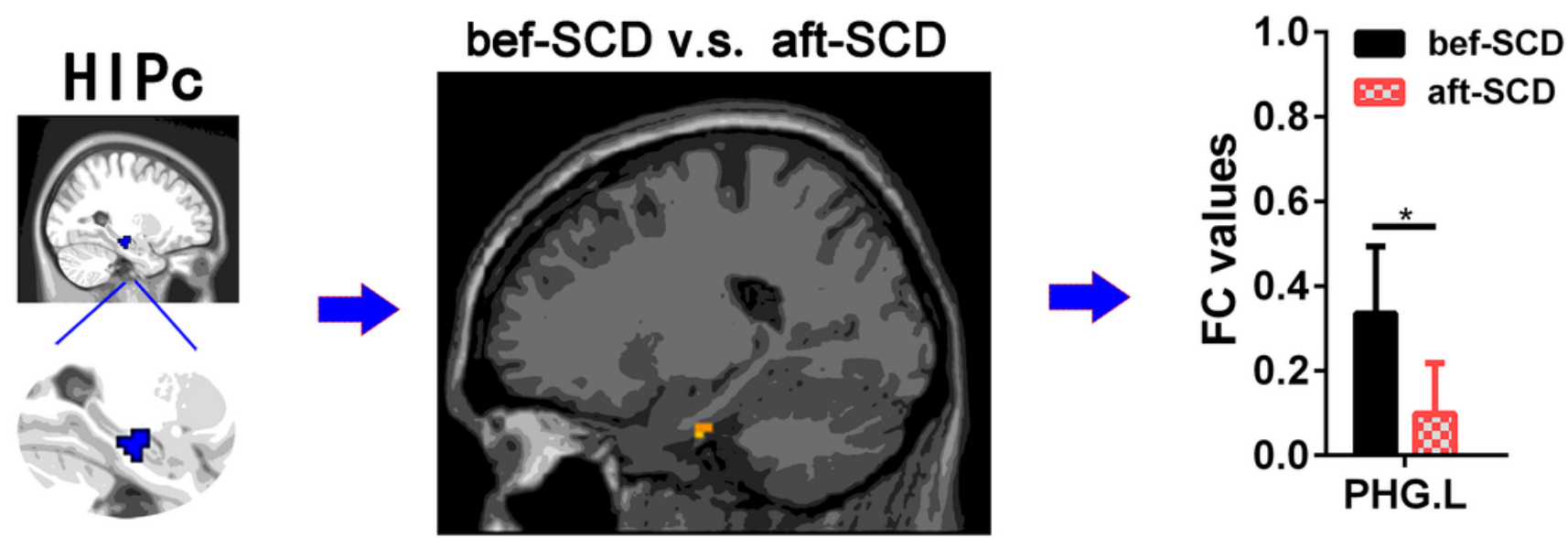

B
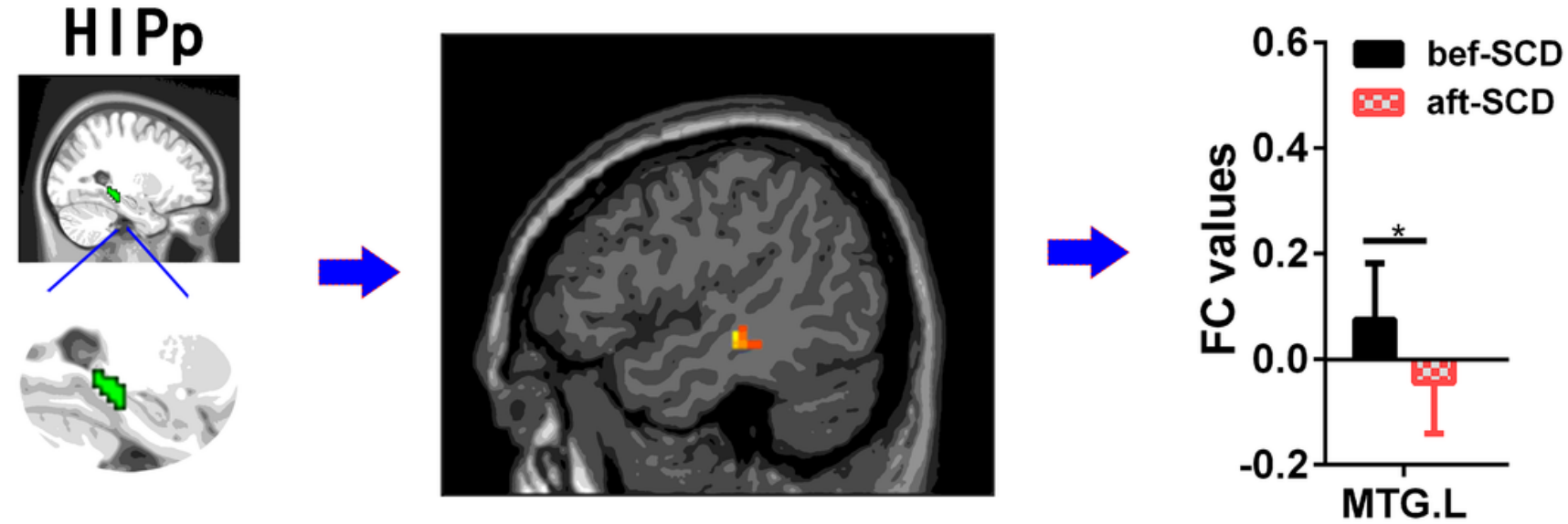

Figure 6

Changes in HIPsub network functional connectivity of SCD before and after 2 weeks of rTMS treatment controlling for age, sex, GM, and education. (A) HIPc seed, brain regions of HIPc functional connectivity changes, and quantitative changes on HIPc functional connectivity of SCD subjects after 2 weeks of rTMS treatment. (B) HIPp seed, brain regions of HIPp functional connectivity changes, and quantitative changes on HIPp functional connectivity of SCD subjects after 2 weeks of rTMS treatment. * $p<0.05$. Abbreviations: bef-SCD, subjective cognitive decline before rTMS treatment; aft-SCD, subjective cognitive decline after rTMS treatment; HIPc, hippocampal cognitive region; HIPp, hippocampal perceptual region; PHG.L, left parahippocampa gyrus; MTG.L, left middle temporal gyrus. 


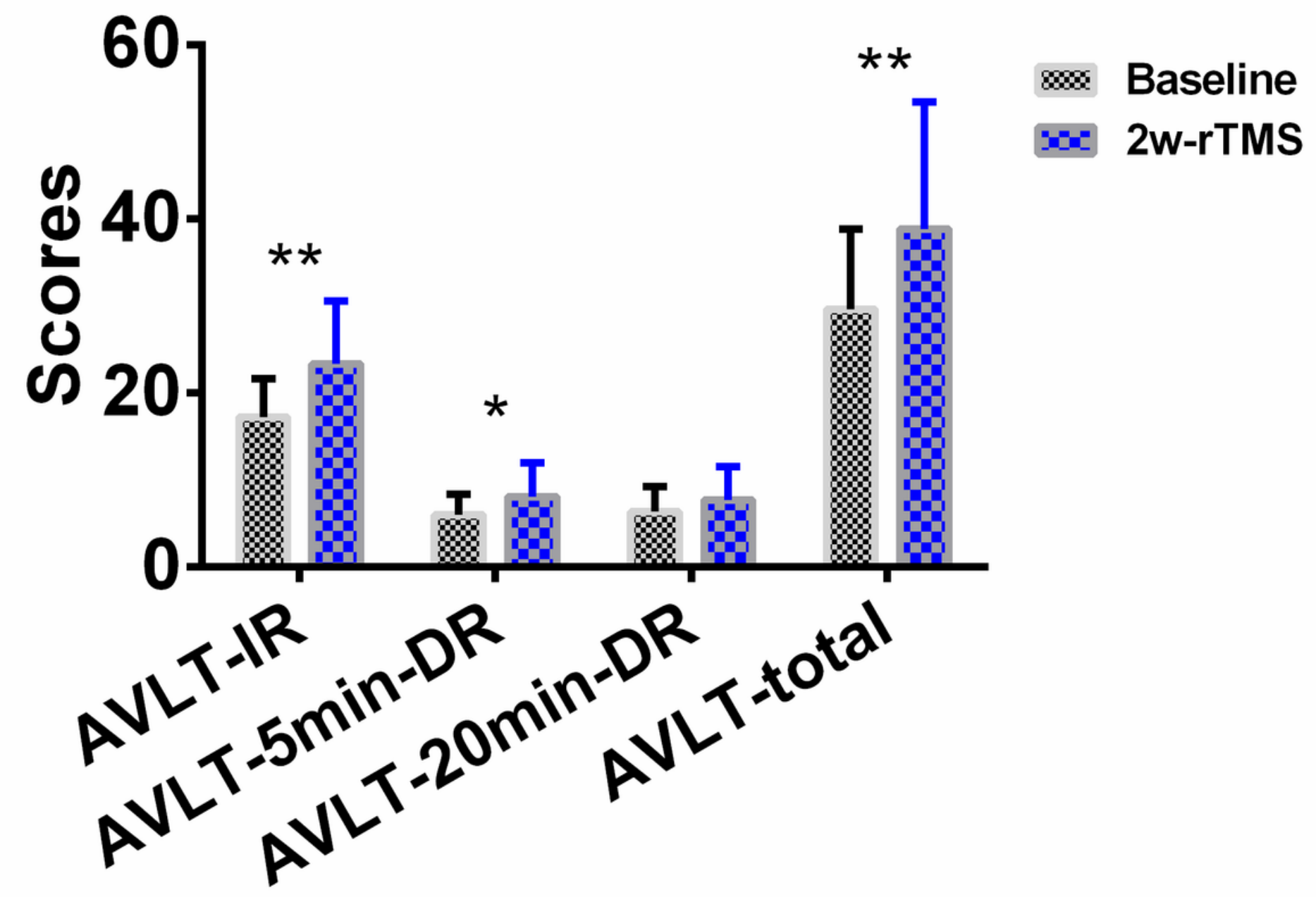

Figure 7

Changes of episodic memory in SCD subjects after 2 weeks of rTMS treatment. Line chart showing the changes in episodic memory in SCD subjects before and after 2 weeks of rTMS treatment. Notably, to improve the statistical power, this study used a re-sampling method of stationary bootstrap $(10,000$ bootstrap samplings) to obtain significance between groups. * $p<0.05,{ }^{*} p<0.01$. Abbreviations: $C N$, healthy controls; SCD, subjective cognitive decline; HIPc, hippocampal cognitive region; MTG.L, left middle temporal gyrus; FC, functional connectivity; 2w-rTMS, 2 weeks of rTMS. AVLT-IR, Auditory Verbal Learning Test - immediate recall; AVLT-5min-DR, Auditory Verbal Learning Test - 5-min delayed recall, AVLT-20min-DR, Auditory Verbal Learning Test -20-min delayed recall.

\section{Supplementary Files}

This is a list of supplementary files associated with this preprint. Click to download.

- Supplementaryinformation.pdf 
- Supplementaryinformation.pdf

Page 25/25 